\title{
Drug Target Interference in Immunogenicity Assays: Recommendations and Mitigation Strategies
}

\author{
Zhandong Don Zhong, ${ }^{1,9}$ Adrienne Clements-Egan, ${ }^{2}$ Boris Gorovits, ${ }^{3}$ Mauricio Maia, ${ }^{4}$ Giane Sumner, \\ Valerie Theobald, ${ }^{6}$ Yuling $\mathrm{Wu}^{7}{ }^{7}$ and Manoj Rajadhyaksha ${ }^{8}$
}

Received 23 March 2017; accepted 11 July 2017; published online 23 October, 2017

\begin{abstract}
Sensitive and specific methodology is required for the detection and characterization of anti-drug antibodies (ADAs). High-quality ADA data enables the evaluation of potential impact of ADAs on the drug pharmacokinetic profile, patient safety, and efficacious response to the drug. Immunogenicity assessments are typically initiated at early stages in preclinical studies and continue throughout the drug development program. One of the potential bioanalytical challenges encountered with ADA testing is the need to identify and mitigate the interference mediated by the presence of soluble drug target. A drug target, when present at sufficiently high circulating concentrations, can potentially interfere with the performance of ADA and neutralizing antibody (NAb) assays, leading to either false-positive or, in some cases, false-negative ADA and NAb assay results. This publication describes various mechanisms of assay interference by soluble drug target, as well as strategies to recognize and mitigate such target interference. Pertinent examples are presented to illustrate the impact of target interference on ADA and NAb assays as well as several mitigation strategies, including the use of anti-target antibodies, soluble versions of the receptors, target-binding proteins, lectins, and solid-phase removal of targets. Furthermore, recommendations for detection and mitigation of such interference in different formats of ADA and NAb assays are provided.
\end{abstract}

KEY WORDS: anti-drug antibody; immunogenicity; mitigation; neutralizing antibody; target interference.

\section{INTRODUCTION}

The ADA and NAb responses have the potential to impact the pharmacokinetic profile, patient safety, and efficacy of biotherapeutics [1-5]. Therefore, it is essential that bioanalytical methods be sufficiently sensitive and highly specific to accurately detect and characterize ADA and NAb responses to these drugs. Numerous publications and industry white papers have been published detailing recommendations for the design, development and validation of ADA

\footnotetext{
${ }^{1}$ Amgen Inc., One Amgen Center Dr., Thousand Oaks, California 91320, USA.

${ }^{2}$ Janssen Research and Development LLC, Janssen Biotherapeutics, Spring House, Pennsylvania, USA.

${ }^{3}$ BioMedicines Design, Pfizer Inc, Andover, Massachusetts, USA.

${ }^{4}$ Genentech, 1 DNA Way, South San Francisco, California, USA.

${ }^{5}$ Regeneron Pharmaceuticals Inc, Tarrytown, New York, USA.

${ }^{6}$ Shire Pharmaceuticals, Lexington, Massachusetts, USA.

${ }^{7}$ MedImmune, Gaithersburg, Maryland, USA.

${ }^{8}$ Regeneron Pharmaceuticals Inc, Tarrytown, New York, USA.

${ }^{9}$ To whom correspondence should be addressed. (e-mail: zhongz@amgen.com)
}

screening, confirmatory, titration, and NAb assays to support the tiered approach generally used for immunogenicity assessments [6-9]. Guidance documents providing regulatory expectations for immunogenicity assessments have also been published by global regulatory agencies [1-3].

The performance of ligand binding assays in complex matrices, such as serum, can be impacted by specific endogenous components interfering with the assay. In ADA and $\mathrm{NAb}$ assays, interference may arise from the presence of circulating drug, an endogenous counterpart of the drug, the drug target, or other serum factors such as rheumatoid factor [7, 10-18]. Just as high levels of drug interfere with ADA detection, the drug target, when present at sufficiently high concentrations in a sample, may also impact the ADA signal or readout and potentially interfere with the detection of clinically relevant ADAs [12-18].

Biotherapeutics exert their biological activity by binding to specific targets, which can then either stimulate or inhibit the pathways related to those targets. Depending on the specific biology of the disease, drug-targeted soluble ligands, shed receptors or receptors that originate from cellular breakdown, may be found in circulation. The presence of these soluble drug targets can lead to erroneous results in the 
ADA measurement, with the amount of targeted ligand or shed receptor reported to correlate with the severity of interference [12-18]. Target interference can present as either false-positive or theoretically false-negative ADA results, depending on the assay format, the concentration of the drug, the level of ADAs, and the affinity and avidity of the overall ADA response. The physico-chemical properties of a soluble drug target and its interactions with the drug under various assay conditions determine the outcome of interference observed. The levels of soluble target ligand or receptor may also fluctuate among individuals and at various sampling time points from the same individual, even within a single disease indication, introducing an additional layer of complexity and variability in ADA assays. Additionally, in some situations, the levels of circulating drug target in a subject may be low and non-interfering at baseline but may increase substantially upon treatment due to the accumulation of drugtarget complexes, either by enhanced shedding of drugengaged cell receptors, increased release of receptors from cellular breakdown, decreased clearance of the drug-target complexes, or perhaps due to feedback mechanisms inherent to the biological pathway. For example, a therapeutic monoclonal antibody (mAb) may sequester a soluble target from its normal degradation pathway, thereby extending the half-life of the inactive target in systemic circulation. When such serum samples are tested in an ADA assay, the target may potentially dissociate from these complexes and achieve sufficient concentrations to cause assay interference. Therefore, it is important to identify appropriate strategies and reagents to mitigate target interference to ensure an accurate immunogenicity assessment [13, 14, 18, 19].

This manuscript provides diverse and relevant examples encompassing target interference observed in both ADA and $\mathrm{NAb}$ assays. These examples provide a framework for the recommendations proposed to predict, assess and mitigate target interference. The recommendations include a decision tree that can be consulted throughout the different phases of the drug development program, as an immunogenicity riskassessment is implemented, and the ADA assay development is undertaken.

\section{OVERVIEW OF ADA ASSAYS}

Several ligand binding assay formats may be used to detect ADAs. The sandwich immunoassay, in which ADAs are captured by a drug-derived reagent and detected with a species-specific detector antibody, could be more tolerant to interference from both circulating drug and drug target [17, 20]. However, this format requires a species-specific positive control ADA, and the detection reagent needs to be characterized to ensure detection of all potential ADA isotypes. Additionally, this assay format is not suitable for detection of human ADAs against a human therapeutic mAb, as the anti-human detector antibody would bind to both the ADAs and the therapeutic. Due to these challenges, the bridging ligand binding assay has emerged as a common format for ADA detection. In a bridging immunoassay, drugderived reagents are typically used to capture and detect ADAs. For example, a biotin-labeled drug can be used as a capture reagent (in conjunction with a streptavidin or avidincoated plate), and a ruthenium-labeled drug used as a detection reagent in an electrochemiluminescence assay platform (Fig. 1a). The drug-derived capture and detection reagents are mixed with the sample. ADAs in the sample can be detected by the formation of a molecular "bridge" between the drug-derived capture reagent and detection reagent. Bridging immunoassays are particularly useful as they can detect the majority of antibody isotypes with the exception of $\mathrm{IgG}_{4}$ in the same assay. Additionally, the same assay format can be adapted across species, and a positive control can be obtained from any species without the need for separate species-specific detection reagents.

The bridging immunoassay format, however, is susceptible to interference primarily due to (1) excess drug that may compete with the drug-derived reagents and reduce the signal of the assay and (2) other non-ADA molecular interactions of serum matrix components that may potentially form a bridge and contribute to the signal in an ADA assay. As this assay format is designed to detect multivalent drug binding, a false-positive result can be generated due to bridging formation mediated by an alternative multi-valent molecule with specific binding with the drug, such as soluble multimeric targets (Fig. 1b). While multimeric soluble drug targets have greater propensity for interference in a bridging assay, it is also theoretically possible for false-negative results to occur if a soluble monomeric target is present in a sufficient quantity to compete out ADAs binding at or near the target-binding epitope (Fig. 1c).

Many biotherapeutics are chronically administered, and the drug serum concentrations may be greater than drug tolerance levels of a standard bridging assay [7]. There are various approaches that have been reported to improve the drug tolerance [20-27]. Although drug interference is outside the scope of this publication, it is worth noting that acid treatment has been frequently exploited to dissociate ADAdrug complexes in a sample to enhance drug tolerance of the assay [24, 26]. Unfortunately, in some cases, these acidic conditions may exacerbate the drug target interference by disrupting drug-target complexes and releasing the accumulated target [14]. Acid treatment has also been reported to multimerize a monomeric target in a sample [28]. The target can then potentially bridge with the capture and detection reagents upon neutralization of the sample $\mathrm{pH}$, leading to false-positive results in the ADA screening assay.

In the tiered approach to ADA analysis, a sample that is screened and classified as potentially positive for ADA is evaluated in a confirmatory assay. In confirmatory assay formats where excess unlabeled drug is added to the sample to inhibit the drug-specific assay signal, the unlabeled drug can also bind to the interfering target. This may result in the inhibition of a "false positive" signal due to multimeric soluble targets, incorrectly "confirming" the "false positive" signal obtained due to target interference. As this type of drug-specific confirmation does not verify the presence of immunoglobulins directed to the drug molecule, the bridging assay format is prone to interference by the drug target. If the soluble drug target does not contain an immunoglobulin domain, an immunodepletion procedure with protein $\mathrm{A} / \mathrm{G} / \mathrm{L}$ can be used to differentiate the binding of a soluble drug target from that of the ADAs [29]; however, this procedure requires significant manipulation of the sample and is prone to causing additional artifacts. Therefore, it may be more 


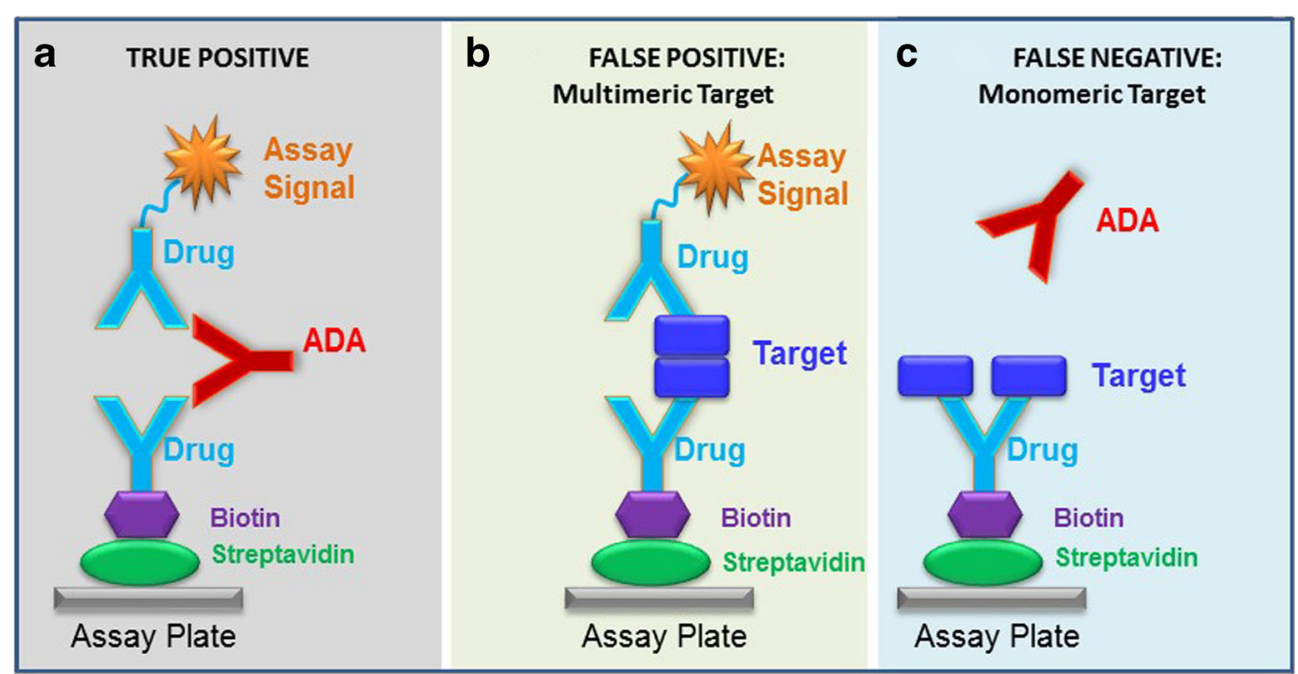

Fig. 1. Interference of drug target in a bridging ADA assay. (a) A Representative example of a true positive signal in the ADA bridging assay resulting from bivalent binding of the ADA to two labeled-drug molecules. (b) A false-positive assay signal arising from multimeric target bridging two labeled-drug molecules. (c) The presence of a molar excess of drug target in a sample, relative to drug, results in target occupying both drug binding sites thus preventing binding of any ADA whose specify is to the drug's targetbinding region, resulting in a false-negative result

efficient to assess the potential for target interference for ADA assays in each biotherapeutic program and, if possible, proactively adopt an alternative mitigation strategy.

To aid in this process, the following sections describe the most commonly used strategies for identifying, overcoming or preventing target interference in ADA assays (Table I).

\section{Competition with Anti-target Antibody}

If an alternative anti-target antibody is available to compete with the drug for binding to target, it may be added to the assay to "scavenge" the drug target during the execution of the ADA bridging assay. It is worth noting that, with the use of anti-target antibodies, it may be necessary to assess whether or not non-neutralizing ADAs are inadvertently removed by the anti-target antibodies. More importantly, if the biotherapeutic is a mAb, the drug and the scavenging antibody should not be conformationally similar, have a similar framework, or share close sequence homology, as any of these attributes may result in cross-reactivity of ADAs to the scavenging anti-target antibody. Therefore, prior to employing an anti-target antibody, it is necessary to assess if non-neutralizing ADAs binding to the non-CDR portions of the drug are inadvertently being masked by the anti-target antibody.

A recently published drug target interference mitigation strategy highlights the importance of selecting competing anti-target antibodies with the appropriate framework [30]. In this example, a bridging assay was developed to detect binding ADAs against a fully human $\mathrm{mAb}$ therapeutic, which achieved its biological activity by binding to a growth factor and thus inhibiting its interaction with the receptor. As high levels of the therapeutic were anticipated in circulation among treated subjects, acid dissociation was employed to increase the drug tolerance of the ADA bridging method. In addition, it was also observed that the levels of the target, known to exist in various multimeric forms, increased in circulation in subjects treated with another biotherapeutic with a similar mechanism of action, resulting in false-positive ADA results in the bridging assay [18]. A human anti-target $\mathrm{mAb}$ that competed with the drug for binding to the target was readily available and tested as a possible reagent to eliminate or mitigate the target interference in the assay. Although this human anti-target $\mathrm{mAb}$ effectively blocked the interference of the drug target, the assay signal of true ADAs was also reduced, due to partial overlapping of the CDRs between the human anti-target $\mathrm{mAb}$ and the therapeutic $\mathrm{mAb}$ (Fig. 2). Thus, it is critical to ensure that competing antitarget $\mathrm{mAbs}$ do not have overlapping sequences that may compete with the drug for ADA binding. Additional human and mouse anti-target mAbs, as well as other target-binding proteins were also evaluated for their ability to block the interference in the presence and absence of the ADA positive control in this study. In the final validated assay design, a recombinant fusion protein was utilized in the neutralization step to inhibit the target interference without affecting detection of ADAs.

\section{Competition with Soluble Receptors for Interfering Ligand}

For biotherapeutics that neutralize ligand-receptor interactions by binding to the ligand (the drug target), the soluble versions of the receptor can be utilized to effectively mitigate target interference, since as the natural binding partner of the ligand, the receptor should have high affinity towards the targeted ligand. As such, the conditions under which the receptor-derived reagents out-compete the drug for target binding are often achievable. In an example where the target of the mAb therapeutic is a multimeric ligand at variable concentrations in drug-naïve samples, the disparity of the target concentration contributed to high variability in the assay response observed in pre-dose samples. Additionally, after drug administration, the level of the target increased due to accumulation of target-drug complexes in circulation. The 
Table I. Summary of target interference mitigation methods

\begin{tabular}{|c|c|c|}
\hline Mitigation methods & Advantages & Disadvantages \\
\hline $\begin{array}{l}\text { Anti-target } \mathrm{mAb}(\mathrm{s}) \text { added to assay } \\
\text { buffer at the screening step }\end{array}$ & $\begin{array}{l}\text { - Greater availability and selection } \\
\text { of mAbs with various affinities } \\
\text { - Ease of production in large quantities } \\
\text { - Seamless integration into the assay } \\
\text { procedure } \\
\text { - No additional sample processing }\end{array}$ & $\begin{array}{l}\text { - Potential removal of ADAs when } \\
\text { having similar conformation, framework } \\
\text { or close sequence homology with the mAb } \\
\text { therapeutic } \\
\text { - Need for high affinity anti-target mAbs or } \\
\text { large quantities of low-affinity mAbs }\end{array}$ \\
\hline $\begin{array}{l}\text { Soluble receptors (for the interfering } \\
\text { ligand) added to assay buffer either } \\
\text { at the screening step or } \\
\text { confirmatory step }\end{array}$ & $\begin{array}{l}\text { - Potentially high affinity towards the } \\
\text { interfering ligand } \\
\text { - Seamless integration into the assay } \\
\text { procedure } \\
\text { - No additional sample processing }\end{array}$ & $\begin{array}{l}\text { - Lack of adequate recombinant version of } \\
\text { the receptor } \\
\text { - High production cost in large quantities } \\
\text { - The recombinant version may be } \\
\text { conformationally different from the native } \\
\text { counterpart } \\
\text { - Stability possibly unsuitable for use as a } \\
\text { reagent }\end{array}$ \\
\hline $\begin{array}{l}\text { Target-specific reagent added to assay } \\
\text { buffer at the confirmation step }\end{array}$ & $\begin{array}{l}\text { - See above } \\
\text { - Lower reagent consumption }\end{array}$ & $\begin{array}{l}\text { - Potential removal of ADAs when } \\
\text { having similar conformation, framework, } \\
\text { or sequence homology with the mAb } \\
\text { therapeutic } \\
\text { - Operationally challenging if high incidence } \\
\text { of screen-positive samples. }\end{array}$ \\
\hline Solid-phase separation of the drug target & $\begin{array}{l}\text { - Does not require high affinity } \\
\text { target-binding reagents }\end{array}$ & $\begin{array}{l}\text { - Potential loss of ADAs due to } \\
\text { additional sample processing step } \\
\text { - Labor intensive and low throughput }\end{array}$ \\
\hline PEG precipitation & - Readily available reagent & $\begin{array}{l}\text { - Potential under-recovery of ADAs } \\
\text { due to additional sample processing step } \\
\text { - Potential impact on ADA stability } \\
\text { - Removal of anti-PEG antibodies } \\
\text { (in pegylated drug molecules) } \\
\text { - Labor intensive and low throughput }\end{array}$ \\
\hline $\begin{array}{l}\text { Therapeutic-specific reagents } \\
\text { (e.g., WGA used to block the } \\
\text { interference of CA148) }\end{array}$ & $\begin{array}{l}\text { - Tailored to specific biotherapeutic } \\
\text { when suitable target-binding } \\
\text { reagents are not available }\end{array}$ & $\begin{array}{l}\text { - Potential impact on ADA detection } \\
\text { unknown } \\
\text { - Need for fully assessing this potential } \\
\text { experimentally }\end{array}$ \\
\hline
\end{tabular}

target was released under the optimized assay conditions, resulting in high assay signals and variability (Fig. 3). A high reported rate of false-positive measurements was thus observed in the ADA assay. No competing anti-target antibodies could be identified to minimize this false-positive target signal. The use of the target receptor alone, at high concentrations, reduced the target signal in a dosedependent manner; however, the receptor alone did not completely abrogate the false-positive signal. With careful consideration of the biochemical properties of the targeted ligand, a mildly acidic $\mathrm{pH}$ was implemented, in conjunction with the receptor at a lower concentration, to further reduce the false-positive signal contributed by the target. With this method the variability observed in both pre- and post-dose samples was effectively managed. Further testing of ADApositive samples from preclinical studies confirmed that the mild acidic conditions had minimal impact on detection of true ADAs.

There can be some limitations in using soluble receptor to minimize drug target interference, however, due to the biophysical and biochemical properties of the soluble version of the receptor. First, sometimes the receptor is a cell surface membrane-bound receptor, and an adequate recombinant soluble extracellular domain may not be readily produced in sufficient quantities to support the bioanalytical work. Secondly, the recombinant version of the target receptor may be conformationally different from its native counterpart. Soluble receptor can also form high molecular weight multimeric complexes that could potentially lead to false-positive ADA signals. Furthermore, depending on the source of production, the costs of purifying the target receptor in required quantities may be prohibitive. Finally, the stability of the recombinant version of the receptor may not be suitable for efficient and long-term use as a critical assay reagent. Nonetheless, if a receptor is available in soluble form without these limitations, it can certainly serve as a good alternative to anti-target antibodies to mitigate target interference.

\section{Use of Target-Binding Reagent with Lower Affinity in the Confirmatory Assay}

It is desired for a competing specific target-binding reagent to have higher affinity to the target than the drug's affinity to the target; however, such high affinity reagents may not be always readily available. Thus, high concentrations of the target-binding reagent may be added to the sample solution, within the assay constraints, regardless of its binding affinity to the target, to ensure that the reagent can effectively 


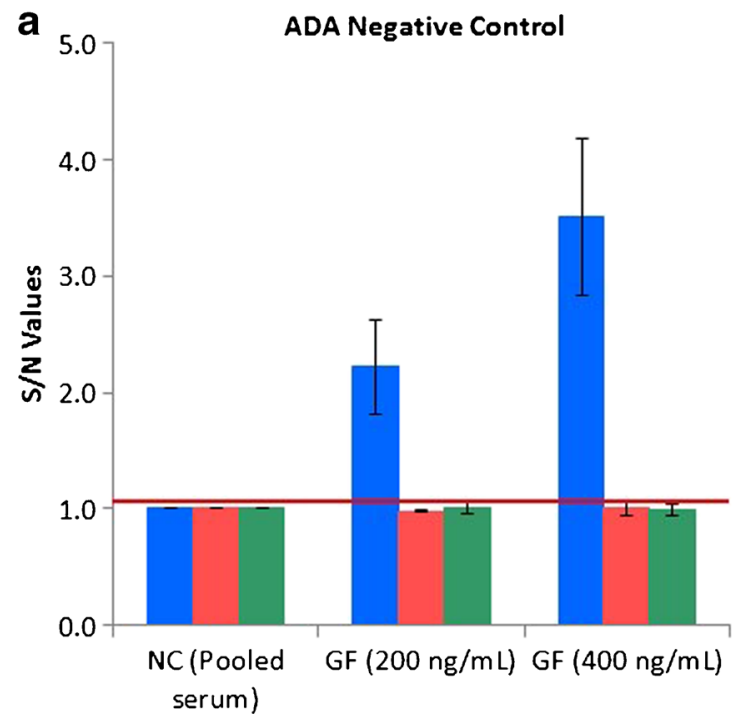

b

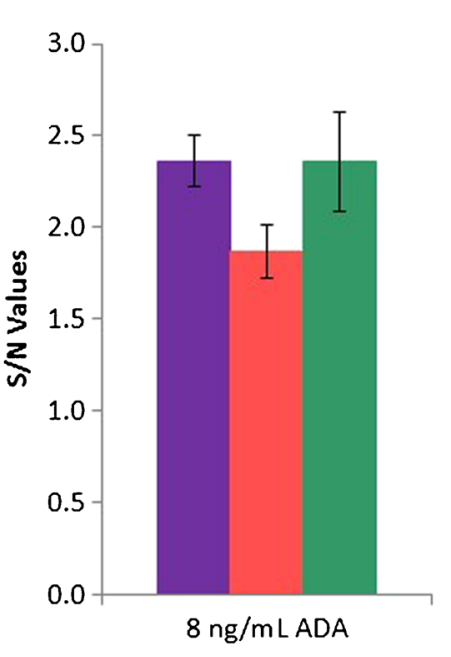

C

High Positive Control

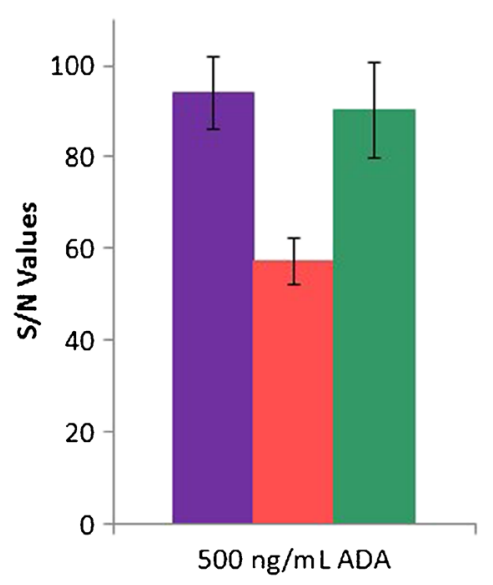

Fig. 2. Partial loss of ADA-positive signal due to use of an inappropriately selected anti-target mAb for blocking target interference. a Target interference by growth factor (GF) was blocked by either a human anti-target mAb $\square$ or a target-binding protein $\square$. b and c ADA signal in the absence of the target $\square$ was reduced by the anti-target mAb due to its amino acid sequence homology with the mAb therapeutic in their CDR regions $\square$. The assay signal was not impacted by target binding protein $\square$. S/N (signal to noise ration) is defined as the assay response over the signal of the negative control

compete out the drug for target binding. If sufficient quantity of this target-binding reagent is available, the reagent can be readily incorporated into both the screen and confirmation binding ADA assays, effectively mitigating the target interference. If this critical reagent is in limited supply, it may be more practical to only incorporate the reagent as part of the confirmation assay. One important consideration of this approach is that it may potentially be challenging in

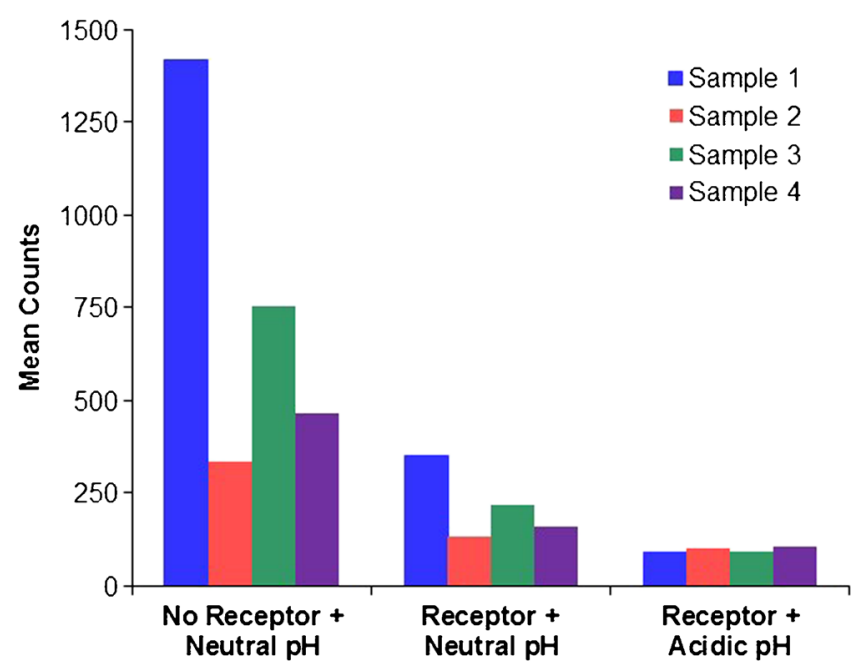

Fig. 3. Blocking target interference with receptor under acidic conditions. Mean counts measured in a bridging ADA assay when four samples from different drug-naïve subjects were analyzed in the absence of target blocking reagent at a neutral $\mathrm{pH}$, or after addition of excess amount of the target receptor either at neutral or acidic assay conditions. Although the presence of the target receptor reduced the false-positive signal caused by target interference, the interference was substantially mitigated when samples were tested under acidic conditions supporting a large clinical study where the rate of confirmation-required samples due to target interference is extremely high in the screening assay.

In an example that represents such a strategy, the therapeutic $\mathrm{mAb}$ binds to a target that is present as a soluble multimer in circulation. The level of the soluble drug target was low at baseline in the drug-naive population; however, it increased after drug administration and resulted in falsepositive responses in the electrochemiluminescent bridging ADA assay. During clinical assay development, a soluble target-specific receptor was selected from a panel of target receptor and anti-target antibodies for its ability to block the false-positive soluble target signal in the assay. A large amount of this receptor reagent would have been required if it was applied to the screening assay due to its lower affinity to the target as compared to the drug. Therefore, this receptor reagent was utilized in the confirmatory assay only. With this strategy, reactive samples identified in the screening assay were evaluated in the second tier confirmation test with the addition of the soluble receptor to the samples tested at a single dilution. False-positive results due to target interference can therefore be identified and removed during the drug-specificity confirmation assay. In this example, drug target produced an assay signal significantly above the background at as low as $800 \mathrm{pg} / \mathrm{mL}$ concentration (Fig. 4). Putative target interference was evaluated during the confirmatory step of the ADA assessment by supplementing serum samples with $5 \mu \mathrm{g} / \mathrm{mL}$ of the receptor reagent aiming to block the target interference and to confirm drug specific ADA detection. Although the lack of noticeable impact of the target-specific treatment on the performance of the ADA assay positive control was demonstrated during assay validation (Fig. 4), the potential impact on ADA measurement was continuously monitored during clinical sample testing. The assay was successfully validated and used to support clinical studies. 


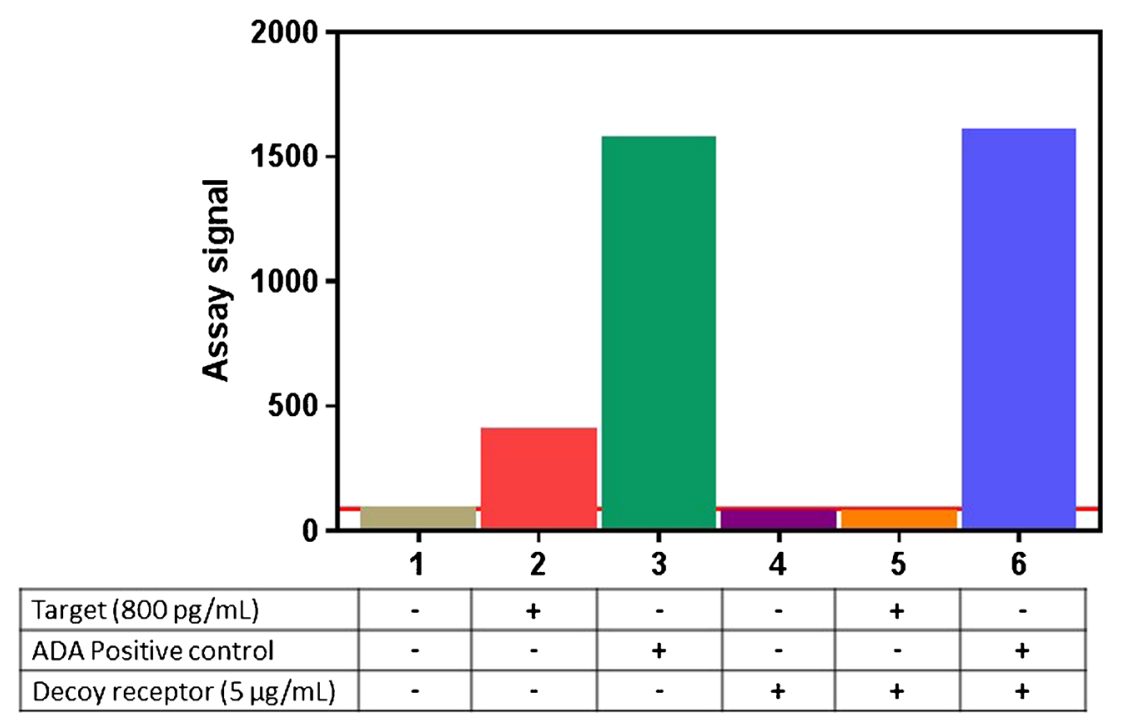

Fig. 4. Investigating putative drug target interference by applying target specific receptor in the confirmatory test. Drug target interference was investigated during the confirmatory step of the ADA assessment. Target specific reagent (decoy receptor) was added to samples at $5 \mu \mathrm{g} / \mathrm{mL}$ to block target interference and to confirm the presence of drug specific ADA. ADA assay positive control performance is not noticeably impacted Red line indicates the signal associated with the negative control

\section{Incorporation of a Solid-Phase Separation Step}

When the affinity of the target-specific reagent available is too low to be used to scavenge the drug target in solution, a solid-phase separation step can be incorporated in the bioanalytical method to remove the interfering target. In this scenario, a target-binding reagent is covalently immobilized on a solid support (e.g., beads or plates), which can be utilized in the assay to remove the interfering target from study samples as discussed in the example below, where the $\mathrm{mAb}$ therapeutic binds to a cell surface receptor that has been shown to shed as soluble dimers in circulation. This was anticipated as a potential interfering factor during the development of the clinical ADA assay. Thus, a mitigation strategy was devised to remove the shed receptor from samples by solid-phase immunodepletion prior to study sample analysis using the bridging ADA assay. Specifically, samples were pretreated with beads that were coated with a target-specific reagent directed to a different target-binding epitope from the drug. In this example, the shed receptors at $800 \mathrm{ng} / \mathrm{mL}$ were adsorbed by the streptavidin nanobeads in combination with a biotinylated target-specific reagent, which were subsequently centrifuged and removed from the sample, allowing the detection of ADAs without target interference (Fig. 5). In spite of the lack of noticeable impact of the solidphase immunoprecipitation treatment on the measurement of the ADA-positive control (Fig. 5), the potential impact on ADA detection was continuously monitored during sample testing. This solid-phase separation approach is generally more labor intensive, with lower throughput than a standard ADA bridging assay. More importantly, it requires additional manipulation of the samples and may potentially result in under-recovery of the ADAs in study samples. Thus, the potential impact of this solid-phase separation method on
ADA detection needs to be carefully assessed during assay development and validation.

\section{Cross-reactivity of Drug Target with Sample Matrix Components}

Erroneous ADA detection could result from potentially complex interactions of the drug target with multiple assay factors and the non-specific sample matrix components. Thus, a careful assessment of potential target interference is warranted in the context of drug target cross-reactivity within the relevant sample matrix under the assay conditions. In the case of the non-bridging sandwich assay format, where an anti-species immunoglobulin detector antibody used to facilitate ADA detection is not expected to bind to the drug and drug target, drug target has been reported to cross-react with the detector antibody indirectly through its interaction with non-specific serum immunoglobulins, resulting in falsepositive ADA measurement [17]. In addition, although utilization of such a detector antibody may offer a possible approach to eliminate false-positive ADA detection, extremely high levels of drug target, regardless of whether it is multimeric or monomeric, could mask ADA detection to give false-negative ADA results by saturating the solid phase where the drug is immobilized for capturing ADAs. For example, a biosensor-based immunoassay was developed to detect ADA against trebananib, an anti-angiogenic Fc fusion protein that reduces angiogenesis by binding to angiopoietin 1 (Ang1) and angiopoietin 2 (Ang2), preventing their interaction with the Tie2 receptor. Ang1 and Ang2 were known to be present as various multimeric forms at low levels among drug-naive subjects; however, the angiopoietin levels increased among drug-treated subjects in phase 1 studies, leading to false-positive ADA detection in the bridging assay [18]. In an attempt to alleviate the interference of both the 


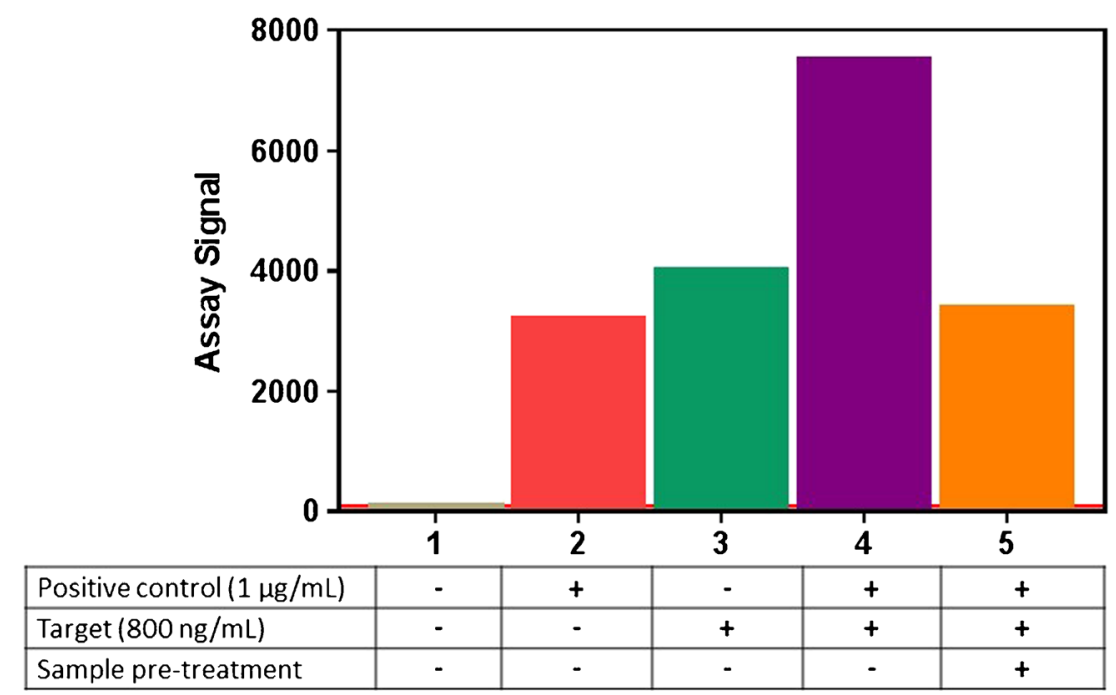

Fig. 5. Removing interfering drug target using a solid-phase separation procedure. Interfering target was removed using an immunoprecipitation procedure. Pretreatment with streptavidin nanobeads in combination with a biotinylated target specific reagent was used to reduce false-positive ADA assay signal generated in the presence of $800 \mathrm{ng} / \mathrm{mL}$ of the target. ADA assay positive control signal remains unaffected. Red line indicates the signal associated with the negative control

drug and the targets, a sandwich assay (biosensor-based) was developed to support ADA testing for late phase clinical studies [17]. In this sandwich assay format, ADAs in a sample were captured by trebananib that was immobilized on the biosensor chip surface (the solid phase). A goat anti-human $\operatorname{IgG}\left(\right.$ Fab' $^{\prime} 2$ with cross-reactivity to $\mathrm{IgG}$, $\operatorname{IgM}$, and $\mathrm{IgA}$ was then injected into the biosensor flow-cell to detect and confirm the captured ADAs (Fig. 6). A humanized antitrebananib $\mathrm{mAb}$ was utilized as the positive control to facilitate the assay development and validation. The detector antibody showed no cross-reactivity to the drug target in protein-free assay buffers at a level that substantially exceeded the highest physiological concentration observed among subjects treated with the drug. When the drug targets were spiked into human serum, however, a dose-response relationship ensued between the concentration of the drug targets and the binding signal of the anti-human detector antibody, leading to false-positive ADA measurement. The above observations suggested that the interference was most likely due to the binding of the soluble drug targets to nonspecific serum immunoglobulins in the samples, which were subsequently detected by the anti-human immunoglobulin detector antibody. The interference was readily mitigated by including a human anti-target $\mathrm{mAb}$ in the sample diluent in the initial sample-binding step for capturing the ADAs. As the captured ADAs were subsequently detected by the antihuman immunoglobulin detector antibody, the human antitarget $\mathrm{mAb}$ was not included in the detection step. In this case, although the drug target was not anticipated to cause interference in the assay, significant target interference was observed due to the interaction of the target with the nonspecific serum matrix components.

\section{Other Potential Mitigation Strategies}

Sometimes a single target blocking reagent is not effective in removing interference from ADA assays. Under such circumstances, it may be necessary to use a "cocktail" of various reagents and assay conditions, including combination of anti-target antibody and receptor [14].

Alternative mitigation strategies could also be devised based on a good understanding of the physico-chemical properties of the target, the drug, and their interactions. For example, in a bridging assay for measuring ADAs against 3A5 TDC, which is an antibody-drug conjugate (ADC) molecule with the antibody domain directed towards the multimeric extracellular domain of CA148, circulating CA148 in serum caused false-positive ADA detection by crosslinking the labeled 3A5 TDC molecules [12]. The assay signal was found to increase with the increase of CA148 in both clinical samples and pooled human serum spiked with commercial CA148. Several anti-CA148 mAbs were evaluated and found to be ineffective in blocking the assay signals resulting from the interference. Since CA148 is a highly glycosylated protein and lectins are known to specifically bind to carbohydrates of glycoconjugates such as CA148, three lectins (WGA, PHA-E, and PHA-M) were evaluated for their ability to block the assay signals arising from binding of CA148 to the labeled 3A5 TDC molecules. The non-specific assay response due to CA148 was significantly suppressed by both WGA and PHA-E, whereas PHA-M had no discernable effect. Interestingly, WGA was the only lectin that was able to block the assay signals resulting from the endogenous CA148 in the serum samples from ovarian cancer patients. Additionally, WGA appeared to block endogenous CA148 more effectively than the commercially available CA148 spiked into the samples. As lectins are known to bind to glycan on human immunoglobulins, the potential impact of the WGA reagent on ADA detection was evaluated. In this example, the WGA reagent was demonstrated to not bind to human immunoglobulins in pooled human serum using a biosensor assay. The interference of circulating CA148 was thus eliminated utilizing WGA, without affecting the ability of the assay to detect relevant ADAs. 


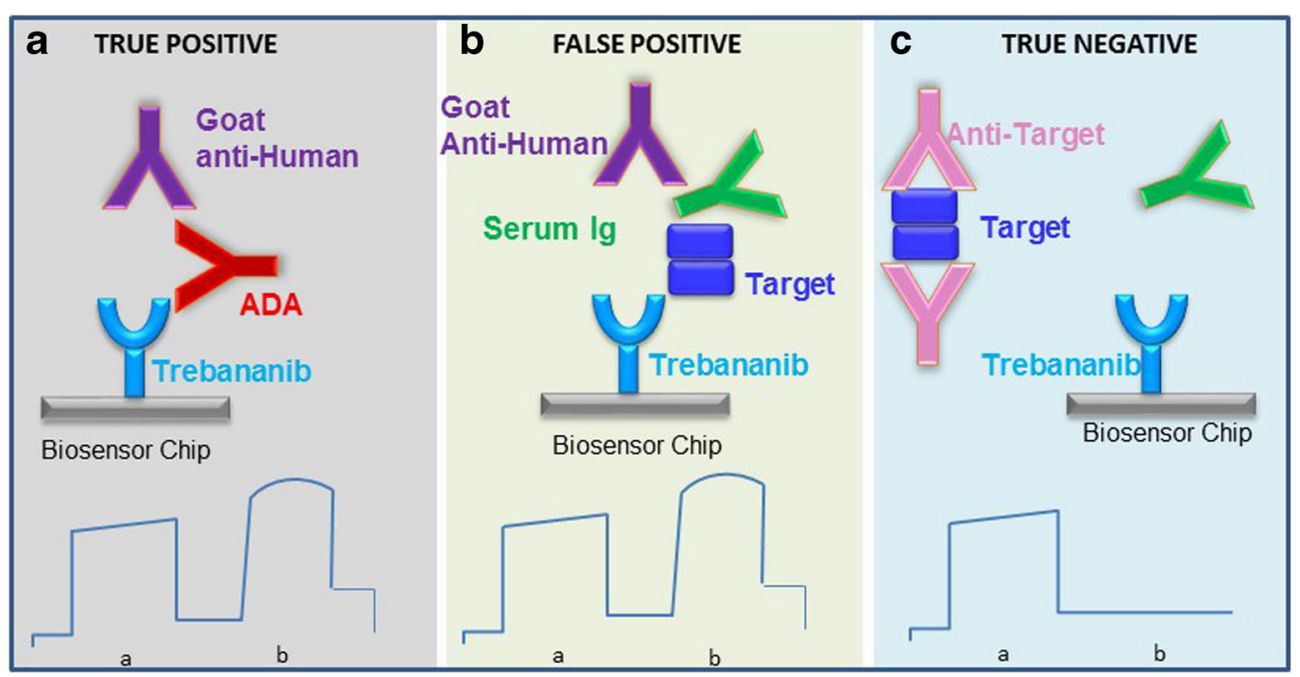

Fig. 6. Drug target interference due to interaction of target with serum matrix immunoglobulins. (a) Biosensor ADA assay with the drug (trebananib) immobilized on the chip. Graph sections: a. ADA binding; b. Anti-human antibody detector binding. (b) False-positive signal caused by binding of the antihuman antibody detector to the complex between target and endogenous non-specific serum immunoglobulin (lg); see "A" above for Graph sections. (c) Mitigations of false-positive signal by addition of anti-target antibody to the sample preventing the target from binding to trebananib; see "A" above for Graph sections

Polyethylene glycol (PEG) precipitation is another method worth consideration. PEGs are highly hydrophilic and are known to attract water molecules away from the solvation layer around the protein, causing protein precipitation. The effectiveness of PEG precipitation greatly depends on the hydrodynamic radius of the protein [31]. In a recent report, a monomeric drug target dimerized at acidic $\mathrm{pH}$ during the acid dissociation step of the bridging assay, leading to false-positive signal [28]. To circumvent the target interference and the low drug tolerance limit, excess drug was added to samples to saturate free ADAs and form drug-ADA complexes. The complexes were then precipitated using PEG 8000, followed by acid dissociation to release the ADAs. Under the acidic conditions, the ADAs were subsequently captured on a solid phase with large surface capacity to allow for specific detection in the bridging assay. PEG precipitation was shown to be a promising method for removing target interference. Other approaches of separating ADAs for detection, such as affinity capture elution (ACE) and solidphase extraction with acid dissociation (SPEAD), which have been demonstrated to improve drug tolerance [21, 32], may also be utilized to mitigate drug target interference. As with the solid-phase separation method (Section 2.4), when applying these sample pretreatment techniques, it is important to ensure that the sample pretreatment does not inadvertently remove low affinity ADA and drug-specific IgM. Additionally, the impact of these pretreatment conditions on ADA stability and recovery needs to be properly evaluated [13].

\section{NAB ASSAYS}

A subset of the ADA responses results in neutralization of drug activity and loss of efficacy. These ADAs are commonly known as neutralizing antibodies (NAbs). If an endogenous homolog of the drug exists, cross-reactive NAbs may also neutralize the endogenous molecule, and in the case of proteins involved in non-redundant pathways, such neutralization can lead to loss-of-function and clinical risk for the patients. Therefore, measurement of NAbs is an important step in assessing the immunogenicity of a biotherapeutic. Two types of assays have been utilized to measure neutralizing activity: functional cell-based bioassays and competitive ligand binding assays.

A functional cell-based NAb assay is typically adapted from a potency assay and may consist of the appropriate responsive cell line, the biotherapeutic, and the sample matrix for NAb detection [6]. The readout of the cell-based assays is generally a biological response such as cell proliferation, apoptosis, or a specific signaling pathway activity. This assay format relies on cells expressing the receptor related to the mechanism of action of the drug. Nonspecific matrix interference in a cell-based NAb assay can be a limitation and can often be eliminated by sample dilution, provided that the assay sensitivity and drug tolerance limit are not adversely impacted. In a cell-based NAb assay, either the drug target (soluble shed receptor) or a competitive antagonist to the drug (ligand) may be present as potential specific interfering factors [33].

As a result of matrix interference, cell-based NAb assays can be highly variable as compared to a non-cell-based competitive binding assay. With a non-cell-based competitive ligand binding assay format, neutralizing activity is detected by measuring the inhibition of the drug's ability to bind to its target. Due to the multi-factorial interactions in this assay format and depending on the levels of the soluble target in the sample, the drug target ligand can result in either false-positive or false-negative NAb detection. For example, a receptor-binding assay was used for detecting NAbs against a fully human antagonistic $\mathrm{mAb}$, which interrupts the ligand-receptor interaction by binding to the ligand (the drug target) [30]. In this NAb assay, the soluble version of the receptor was immobilized on the solid phase. The interaction of the labeled ligand with its receptor was blocked by the drug binding to the ligand (Fig. 7a). In the presence of NAb, the binding of the labeled ligand to the receptor was restored, generating an increased assay 


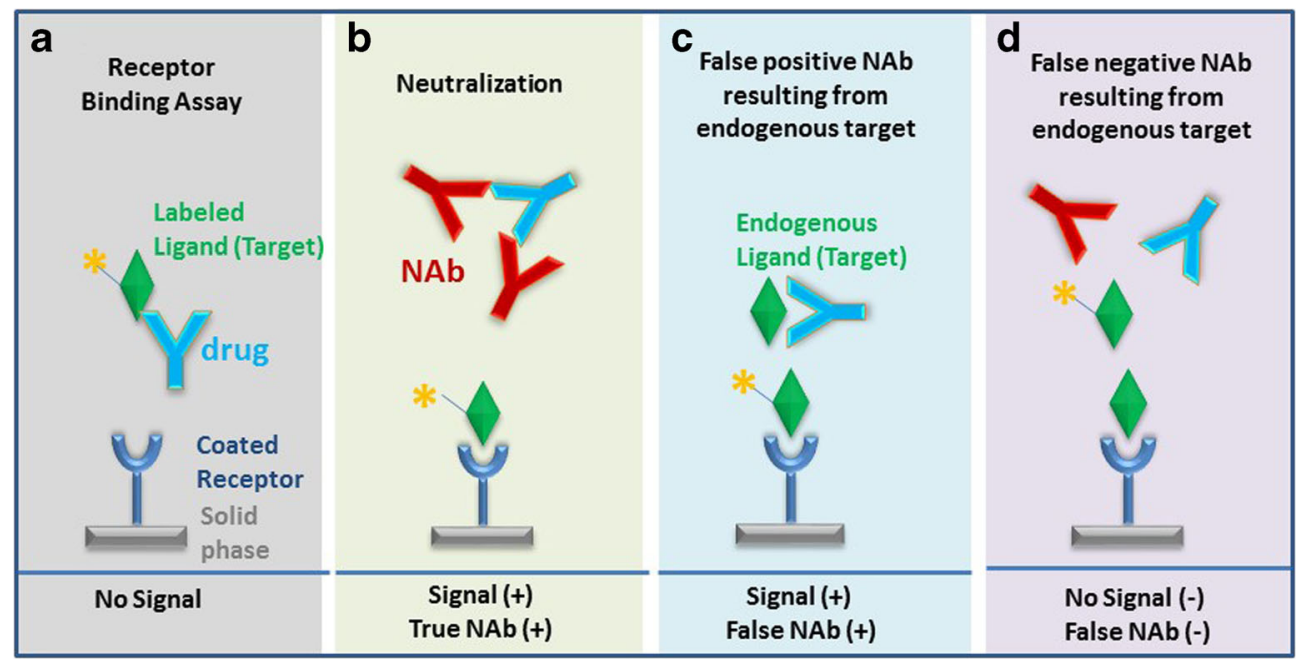

Fig. 7. Drug target interference in receptor-binding NAb assay. NAb assay diagram: drug blocks binding of labeled ligand (drug target) to its receptor, which has been immobilized on a solid phase (a). A true-positive signal due to NAb binding to the drug, preventing it from binding to the labeled drug target (b). A falsepositive neutralization signal due to binding of endogenous target with the drug, preventing drug binding to the labeled target (c). False-negative NAb result due to endogenous target occupying the immobilized receptor $(\mathbf{d})$

readout (Fig. 7b). Figure 8 shows that the presence of high level of the unlabeled endogenous target initially led to false-positive NAb measurement as the endogenous target interfered with the binding between the drug and the labeled ligand used in the assay (Fig. 7c). Interestingly, when the level of the endogenous target approached the highest concentrations that were projected post dosing in clinical trial, a false-negative signal was observed in the NAb assay, as the endogenous target started blocking the interaction of the labeled ligand with the receptor by occupying the binding site on the receptor coated on the solid phase (Fig. 7d). Since NAb assays are prone to drug interference leading to false-negative readouts, such a false-negative readout due to target interference may be overlooked.

\section{SUMMARY AND RECOMMENDATIONS}

The presence of soluble drug targets can interfere with the detection of ADAs, confounding the correlation of immunogenicity and its impact on the PK/PD, safety, and efficacy of a biotherapeutic. Drug target interference is greatly dependent on the biochemical and biophysical properties of the target, the specific pharmacological pathway associated with the target, the mechanism of action of the drug, and the relative affinity between the drug and the endogenous target. The outcome of the interference can also be impacted by the assay design and the multi-factorial interactions that can potentially take place between the drug, drug target, non-specific matrix factors, and ADAs.

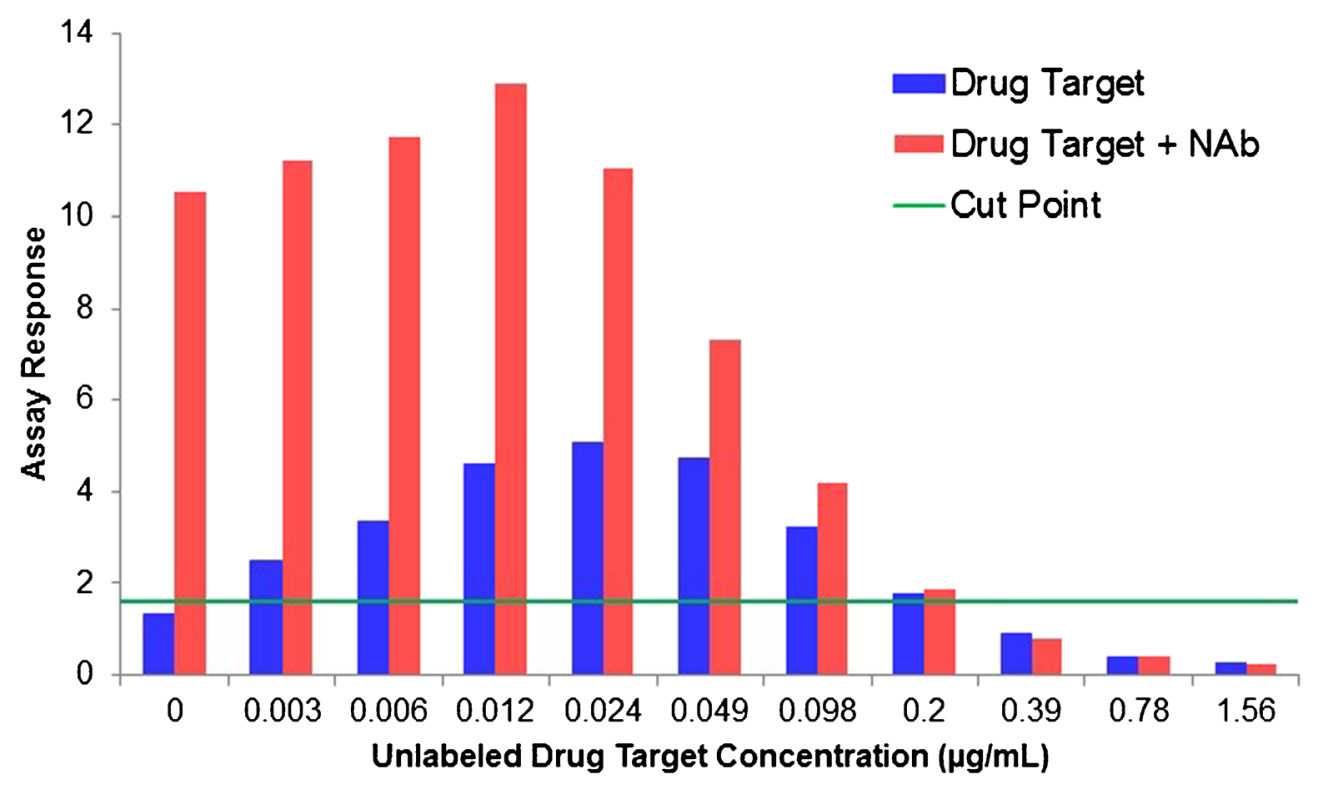

Fig. 8. Drug target can cause either false-positive or false-negative NAb detection. The presence of low levels of drug target caused false positive NAb detection $\square$. The presence of higher levels $(>0.2 \mu \mathrm{g} / \mathrm{mL})$ of drug target resulted in false-negative NAb measurement 
Therefore, it is imperative to proactively identify, assess, and mitigate target interference, during assay development and validation, with a risk-based approach, to ensure that an appropriate assessment of the immunogenicity of a given biotherapeutic can be conducted.

Drug targets can be either soluble or cell membrane-bound. Soluble drug targets can be either secreted or shed from cell surfaces. While soluble multimeric targets (even at low concentrations) can lead to false-positive results in an ADA assay, sufficiently high levels of monomeric targets can also potentially mask the detection of ADAs that may bind at or near the target binding site, leading to false negative results. Further, during treatment phase the drug target can accumulate at high levels and can be complexed with the drug, which could exacerbate the interference under the assay conditions. Similar to ADA assays, $\mathrm{NAb}$ assays can also be susceptible to target interference. The outcome of target interference in a NAb assay, regardless of the target's multimerization state, can lead to either false-positive or false-negative results depending on the assay format and the levels of the drug target in a sample at a particular time point in the study due to the complex mechanics of a NAb assay. Target interference can also contribute to insufficient sensitivity and greater variability in both ADA and NAb assays. Utilization of the design of experiments approach should greatly facilitate identification of target interference and improve overall assay performance.

A decision tree has been developed to provide general guidance for identifying target interference and developing strategies to mitigate the interference (Fig. 9).

1. For soluble targets, one of the challenges in assessing and mitigating drug target interference is that the relevant levels of the drug target in a given population are often unknown prior to the clinical study. Therefore, whenever possible, an effort should be made to determine the total drug target concentrations in the population of interest early in a drug development program [34].

2. With soluble drug targets and their interactions with other non-specific serum components in the ADA and NAb assays, target interference can be observed even prior to drug administration. Efforts should be made to differentiate such target interference from pre-existing antibodies against the biotherapeutic during assay development and validation. Target interference may increase as target concentrations increase after drug treatment due to the formation and accumulation of target-drug complexes, especially if an acid dissociation step has been incorporated into the ADA assay to enhance drug tolerance. It is therefore important that a target interference assessment be performed during assay development, using target levels in excess of those reported in the literature (or measured) for the specific disease indication, to account for the possible accumulation of the target in postdose samples. If high target levels are not expected, or are not sufficiently high to interfere in the ADA and NAb assays, no additional considerations may be necessary.

3. If the outcome of the target interference assessment shows false-negative or false-positive results, irrespective of monomeric or multimeric targets, it is recommended that an effective mitigation strategy be developed early on, to ensure that true ADA responses are detected in postdose as well as predose samples.

4. Furthermore, if target concentrations can lead to assay interference, it is recommended that targetbinding reagents be developed and utilized to characterize the potential impact of target interference on ADA detection, so an effective strategy can be developed and implemented to mitigate the interference.

5. Once the risk of impactful drug target interference in an ADA or NAb assay has been identified, a variety of approaches, ranging from the simple addition of targetblocking reagents to more complex assay modifications, can be explored. Anti-target antibodies, soluble receptors, target-binding proteins, and lectins have been reported to effectively eliminate target interference in ADA assays, with several examples discussed in this publication.

6. Since drug target is employed in a typical NAb assay [35], the mitigation of drug target interference in $\mathrm{NAb}$ assays remains challenging. While the screening and confirmatory ADA assay can be the first line of defense against target interference, protein $\mathrm{A} / \mathrm{G} / \mathrm{L}$ depletion can be used to confirm detection of immunoglobulins in a NAb assay.

7. Various sample pretreatment techniques have also been reported for successful removal of soluble drug targets; however, the impact of these pretreatment conditions on ADA stability and recovery needs to be properly evaluated. Therefore, a careful evaluation of potential target-blocking reagents early in a program, taking into account their specificity, availability, ease of production, cost, etc., may yield a readily implemented mitigation strategy. In addition, the known physico-chemical properties of the target can also be explored to develop other sample treatment conditions or assay conditions that specifically interrupt the binding of the target to the drug, while maintaining the drug binding properties of ADAs.

8. Once a strategy has been devised to mitigate target interference, it is essential to evaluate its effectiveness using actual clinical samples, with different levels of the target, since results obtained during assay development, generally conducted with recombinant versions of the soluble target, may not reflect results observed with the native target present in circulation.

9. It is important to acknowledge that ADA-positive controls used in the assessment of various mitigation methods are surrogate samples, often not fully representing the variety of ADA molecules that can be found as a result of immune responses. Thus, the outcome of each assessment may, in certain cases, not be predictive of what will take place when study samples are tested.

10. Independent of the strategy implemented, it is critical to ensure that the ability of the assay to detect all ADAs of various affinities, isotypes, and subclasses has not been affected. For mAb therapeutics, it is 


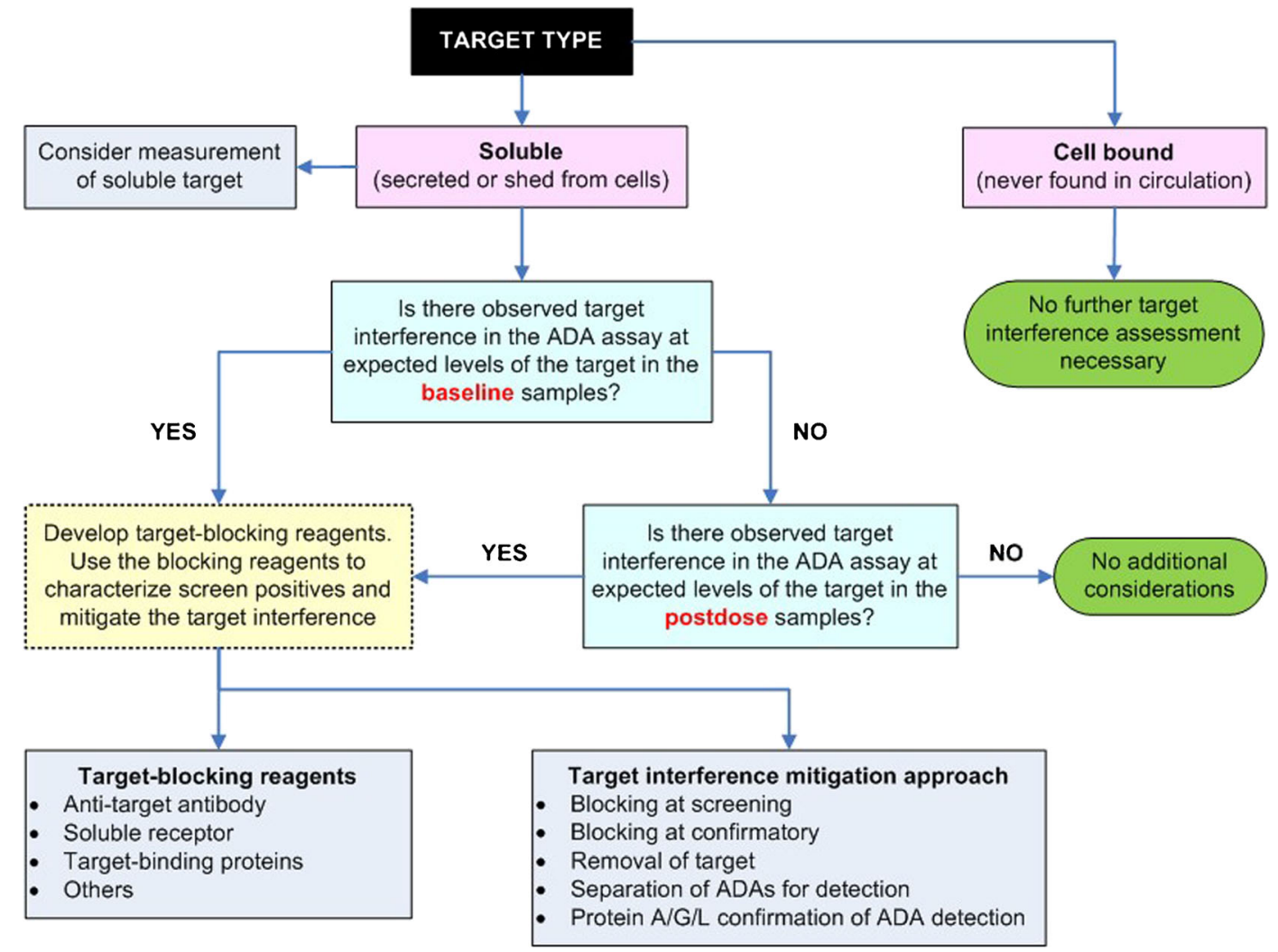

Fig. 9. Decision tree for identifying and mitigating drug target interference. This chart provides suggestions for most typical target interference situations. It is not intended to accommodate all possible interference scenarios

important to ensure that a competing alternative antitarget $\mathrm{mAb}$ has a different $\mathrm{CDR}$ and if possible, framework from the therapeutic mAb. This will ensure that the detection of relevant ADAs is not inadvertently obscured by the target-blocking reagent.

11. It is also highly recommended that the rate of positive ADA measurement be examined in the context of the disease population and the nature of the drug during clinical studies to ensure that the positive binding is indeed due to ADAs [18], regardless of the assay formats or the target interference mitigation strategies employed. For example, a high incidence of ADA formation would not be expected in a cancer population, where chemotherapy is co-administered with a therapeutic $\mathrm{mAb}$. Rather, such positive assay responses may be indicative of target binding rather than ADA measurement, because the subjects may be immunecompromised due to ablative therapies. Therefore, it is critical to take a holistic approach to assess potential drug target interference, taking into consideration both the literature about the therapeutic target, the relevant serum factors, and the relevant clinical setting.

In summary, the presence of soluble drug target interference in study samples may lead to considerable bioanalytical challenges in the context of immunogenicity data interpretation. A clear understanding of the physio-chemical properties of both the therapeutic and its soluble target, as well as their interaction can provide valuable insight to generate and select the best reagents to mitigate the target interference, and also to select the appropriate assay format and conditions. Therefore, it is essential to understand the biology of both the biotherapeutic and the target to devise effective strategies to identify and mitigate drug target interference, so the immunogenicity of given therapeutic can be accurately assessed.

\section{ACKNOWLEDGMENTS}

The authors thank Eric Wakshull, PhD, of Genentech for his critical review of this manuscript; and Alok Rathi and Qiang Qu, both of EMD Serono, for providing data and useful discussions.

\section{COMPLIANCE WITH ETHICAL STANDARDS}

Disclaimer The contents of this article reflect the personal opinions of the authors and may not represent the official positions or perspectives of their affiliated organizations.

Open Access This article is distributed under the terms of the Creative Commons Attribution 4.0 International License (http://creativecommons.org/licenses/by/4.0/), which permits unrestricted use, distribution, and reproduction in any medium, provided you give appropriate credit to the original author(s) and the source, provide a link to the Creative Commons license, and indicate if changes were made. 


\section{REFERENCES}

1. US Food and Drug Administration (FDA) (2014) Guidance for Industry. Immunogenicity assessment for therapeutic protein products. Published as a notice in the Federal Register: 79 Fed. Reg. 47649, August 14, 2014. Docket No. FDA-2013-D-0092.

2. Committe for Medicinal Product for Human Use (CHMP) (draft) Guideline on immunogenicity assessment of biotechnologyderived therapeutic proteins (draft guidance). Doc. Ref. EMEA/CHMP/BMWP/14327/2006, London, 2016.

3. US Food and Drug Administration (FDA) (2016) Guidance for industry: assay development forimmunogenicity testing of therapeutic proteins (draft guidance). Published as a notice in the Federal Register: 81 FR 24106, April 2016. Docket No. FDA-2009-D-0539.

4. Buttel IC, Chamberlain P, Chowers Y, Ehmann F, Greinacher A, Jefferis R, et al. Taking immunogenicity assessment of therapeutic proteins to the next level. Biologicals: J Int Assoc Biol Stand. 2011;39(2):100-9.

5. Koren E, Smith HW, Shores E, Shankar G, Finco-Kent D, Rup $\mathrm{B}$, et al. Recommendations on risk-based strategies for detection and characterization of antibodies against biotechnology products. J Immunol Methods. 2008;333(1-2):1-9.

6. Gupta S, Devanarayan V, Finco D, Gunn GR 3rd, Kirshner S, Richards S, et al. Recommendations for the validation of cellbased assays used for the detection of neutralizing antibody immune responses elicited against biological therapeutics. J Pharm Biomed Anal. 2011;55(5):878-88.

7. Wang YM, Fang L, Zhou L, Wang J, Ahn HY. A survey of applications of biological products for drug interference of immunogenicity assays. Pharm Res. 2012;29(12):3384-92.

8. Mire-Sluis AR, Barrett YC, Devanarayan V, Koren E, Liu H, Maia M, et al. Recommendations for the design and optimization of immunoassays used in the detection of host antibodies against biotechnology products. J Immunol Methods. 2004;289(1-2):1-16.

9. Shankar G, Devanarayan V, Amaravadi L, Barrett YC, Bowsher R, Finco-Kent D, et al. Recommendations for the validation of immunoassays used for detection of host antibodies against biotechnology products. J Pharm Biomed Anal. 2008;48(5):1267-81.

10. Araujo J, Zocher M, Wallace K, Peng K, Fischer SK. Increased rheumatoid factor interference observed during immunogenicity assessment of an Fc-engineered therapeutic antibody. J Pharm Biomed Anal. 2011;55(5):1041-9.

11. Tatarewicz S, Miller JM, Swanson SJ, Moxness MS. Rheumatoid factor interference in immunogenicity assays for human monoclonal antibody therapeutics. J Immunol Methods. 2010;357(1-2):10-6.

12. Carrasco-Triguero M, Mahood C, Milojic-Blair M, Amaya C, Ruppel J, Hong K, et al. Overcoming soluble target interference in an anti-therapeutic antibody screening assay for an antibodydrug conjugate therapeutic. Bioanalysis. 2012;4(16):2013-26.

13. Chen K, Page JG, Schwartz AM, Lee TN, DeWall SL, Sikkema DJ, et al. False-positive immunogenicity responses are caused by $\mathrm{CD} 20+\mathrm{B}$ cell membrane fragments in an antiofatumumab antibody bridging assay. J Immunol Methods. 2013;394(1-2):22-31.

14. Dai S, Schantz A, Clements-Egan A, Cannon M, Shankar G. Development of a method that eliminates false-positive results due to nerve growth factor interference in the assessment of fulranumab immunogenicity. AAPS J. 2014;16(3):464-77.

15. Mikulskis A, Yeung D, Subramanyam M, Amaravadi L. Solution ELISA as a platform of choice for development of robust, drug tolerant immunogenicity assays in support of drug development. J Immunol Methods. 2011;365(1-2):38-49.

16. Schwickart M, Mehrzai F, Pearson J, Shaghasi N, Chavez C, Schneider A, et al. Identification and elimination of targetrelated matrix interference in a neutralizing anti-drug antibody assay. J Immunol Methods. 2014;403(1-2):52-61.

17. Weeraratne DK, Lofgren J, Dinnogen S, Swanson SJ, Zhong ZD. Development of a biosensor-based immunogenicity assay capable of blocking soluble drug target interference. J Immunol Methods. 2013;396(1-2):44-55.
18. Zhong ZD, Dinnogen S, Hokom M, Ray C, Weinreich D, Swanson SJ, et al. Identification and inhibition of drug target interference in immunogenicity assays. J Immunol Methods. 2010;355:21-8.

19. Liao K, Meyer E, Lee TN, Loercher A, Sikkema D. Inhibition of interleukin-5 induced false positive anti-drug antibody responses against mepolizumab through the use of a competitive blocking antibody. J Immunol Methods. 2017;441:15-23.

20. Li J, Schantza A, Schwegler M, Shankar G. Detection of lowaffinity anti-drug antibodies and improved drug tolerance in immunogenicity testing by Octet ${ }^{\circledR}$ biolayer interferometry. J Pharm Biomed Anal. 2011;54:286-94.

21. Bourdage JS, Cook CA, Farrington DL, Chain JS, Konrad RJ. An affinity capture elution (ACE) assay for detection of antidrug antibody to monoclonal antibody therapeutics in the presence of high levels of drug. J Immunol Methods. 2007;327(1-2):10-7.

22. Lofgren JA, Wala I, Koren E, Swanson SJ, Jing S. Detection of neutralizing anti-therapeutic protein antibodies in serum or plasma samples containing high levels of the therapeutic protein. J Immunol Methods. 2006;308(1-2):101-8.

23. Neubert H, Grace C, Rumpel K, James I. Assessing immunogenicity in the presence of excess protein therapeutic using immunoprecipitation and quantitative mass spectrometry. Anal Chem. 2008;80(18):6907-14.

24. Patton A, Mullenix MC, Swanson SJ, Koren E. An acid dissociation bridging ELISA for detection of antibodies directed against therapeutic proteins in the presence of antigen. J Immunol Methods. 2005;304(1-2):189-95.

25. Rispens T, Hart MH, Ooijevaar-de Heer P, van Leeuwen A, Vennegoor A, Killestein J, et al. Drug interference in immunogenicity assays depends on valency. J Pharm Biomed Anal. 2013;85:179-85.

26. Sickert D, Kroeger K, Zickler C, Chokote E, Winkler B, Grenet $\mathrm{JM}$, et al. Improvement of drug tolerance in immunogenicity testing by acid treatment on Biacore. J Immunol Methods. 2008;334(1-2):29-36.

27. Smith HW, Butterfield A, Sun D. Detection of antibodies against therapeutic proteins in the presence of residual therapeutic protein using a solid-phase extraction with acid dissociation (SPEAD) sample treatment prior to ELISA. Regul Toxicol Pharmacol: RTP. 2007;49(3):230-7.

28. Zoghbi J, Xu Y, Grabert R, Theobald V, Richards S. A breakthrough novel method to resolve the drug and target interference problem in immunogenicity assays. J Immunol Methods. 2015;426:62-9.

29. Sanchez S, Barger T, Zhou L, Hale M, Mytych D, Gupta S, et al. Strategy to confirm the presence of anti-erythropoietin neutralizing antibodies in human serum. J Pharm Biomed Anal. 2011;55(5):1265-74.

30. Jacques S, Lee S, Shalini G, Zhong ZD. Considerations in Mitigating Drug Target Interference in Immunogenicity Testing. AAPS Annual Meeting. Orlando, FL. 2015. Abstract R6218.

31. Sim SL, He T, Tscheliessnig A, Mueller M, Tan RB, Jungbauer A. Protein precipitation by polyethylene glycol: a generalized model based on hydrodynamic radius. J Biotechnol. 2012;157(2):315-9.

32. Butterfield AM, Chain JS, Ackermann BL, Konrad RJ. Comparison of assay formats for drug-tolerant immunogenicity testing. Bioanalysis. 2010;2:1961-9.

33. Wu Y, Li JJ, Kim HJ, Liu X, Liu W, Akhgar A, et al. A neutralizing antibody assay based on a reporter of antibodydependent cell-mediated cytotoxicity. AAPS J. 2015;17(6):141726.

34. Lee JW, Kelley M, King LE, Yang J, Salimi-Moosavi H, Tang MT, et al. Bioanalytical approaches to quantify "total" and "free" therapeutic antibodies and their targets: technical challenges and PK/PD applications over the course of drug development. AAPS J. 2011;13(1):99-110.

35. Wu B, Chung S, Jiang XR, McNally J, Pedras-Vasconcelos J, Pillutla R, et al. Strategies to determine assay format for the assessment of neutralizing antibody responses to biotherapeutics. AAPS J. 2016. 